\title{
EFFECT OF SILYMARIN ON INTESTINAL ALKALINE PHOSPHATASE LEVEL IN A RAT MODEL OF TYPE 2 DIABETES MELLITUS: STREPTOZOTOCIN AND HIGH-FAT DIET TREATED WISTAR RATS
}

\author{
LALITHA V*, SIVAKUMAR T
}

Department of Pharmacology, Nandha College of Pharmacy and Research Institute, Erode, Tamil Nadu, India. Email: sasiikv@gmail.com Received: 20 June 2018, Revised and Accepted: 13 July 2018

\section{ABSTRACT}

Objective: This research elucidated the role of silymarin on intestinal alkaline phosphatase (IAP) level in type 2 diabetic rats.

Methods: The type 2 diabetes mellitus was induced by a high-fat diet (HFD - 58\% calories fat) for 2 weeks, and rats were intraperitoneally injected with streptozotocin (STZ) $35 \mathrm{mg} / \mathrm{kg}$. Wistar rats were divided into four groups. Group I served as a non-diabetic (normal), Group II served as diabetic, Group III diabetic animals treated glibenclamide $600 \mu \mathrm{g} / \mathrm{kg}$ for 14 days, and Group IV diabetic animal treated with glibenclamide and silymarin $50 \mathrm{mg} / \mathrm{kg} /$ twice $/ \mathrm{d}$ for 14 days. At the end of the study, blood glucose, lipid profile, and IAP level were measured.

Results: A significant decrease in IAP, elevated levels of blood glucose, and lipid profile was seen in diabetic rats when compared with normal. The silymarin treatment showed a significant increase in IAP level, a significant reduction in glucose and lipid profile than diabetic rats.

Conclusion: The present study concludes that silymarin treatment enhances the IAP levels which protect against hyperglycemia, hyperlipidemia, and vascular complications in diabetic rats.

Keywords: High-fat diet, Streptozotocin, Silymarin, Intestinal alkaline phosphatase.

(C) 2018 The Authors. Published by Innovare Academic Sciences Pvt Ltd. This is an open access article under the CC BY license (http://creativecommons. org/licenses/by/4. 0/) DOI: http://dx.doi.org/10.22159/ajpcr.2018.v11i11.28008

\section{INTRODUCTION}

Diabetes mellitus is a chronic disorder characterized by high blood glucose level, insulin resistance, and relative insulin deficiency $[1,2]$. Type 2 diabetes mellitus (T2DM) is more common, because of increases in unhealthy food, sedentary lifestyle, and obesity. In later, it developed as vascular, neuropathic complications, dysfunction, and failure of different organs, especially the kidneys, nerves, heart, and blood vessels [3-5]. Diabetic management very challenges to patients and health-care systems. Urgently, we require the best choice of treatment to reduce the development and progression of diabetes and its complications. The high-fat diet (HFD) and streptozotocin (STZ) were an ideal model for T2DM because it mimics to metabolic characteristics of human T2DM.

Silymarin is flavonolignan, obtained from seeds of Silybum marianum L. as medical remedies since the time of ancient Greece. The major active constituents are silybin, silychristin, and silydianin, collectively called silymarin [6]. It is an antioxidant, hepatoprotective, antidiabetic, antihyperlipidemic, anti-inflammatory, antifibrotic, and cytoprotective [7,8]. It improves the glycemic control and reduces the diabetic complications. Silymarin decreases the triglycerides, very low-density lipoprotein (VLDL) synthesis, and the availability of free VLDL secretion in the intestine. It reduces lipid accumulation by decreasing adipogenic factors such as fatty acid-binding protein 4 and peroxisome proliferator-activated receptor $\gamma$ [9].

The intestinal microbiota is a pivotal role in maintaining human health and well-being. Intestinal alkaline phosphatase (IAP) is an important brush border enzyme expressed an entire part of the intestine with the highest in the duodenum and produces maximal activity in ileum [10]. IAP plays an important role in maintain gut homeostasis. IAP regulates intestinal $\mathrm{pH}$, detoxifies bacterial lipopolysaccharide [11] and binds to intracellular proteins, and decreases the movement of the fat droplet across the enterocyte. The IAP plays a crucial role in decreasing the rate of fat absorption. IAP expression is modulated by several nutrition substances [12]. IAP is a protective role against hyperlipidemia, T2DM, endotoxemia, and metabolic syndrome [13].

Several studies reported the antidiabetic and antihyperlipidemic activity of silymarin mainly due to its antioxidant effect and decreased synthesis of lipids. IAP supplementation can protect against T2DM. Hence, we evaluate the effect of silymarin on IAP levels and their role in T2DM diabetes.

\section{METHODS}

Chemicals

Silymarin was obtained from Sigma-Aldrich Chemical Pvt., Limited, India. All other drugs and chemicals used in the study were obtained commercially and were of analytical grade.

\section{Experimental animals}

The Wistar rats (200-250 g) were maintained in clean, sterile, and polypropylene cages with paddy husk as bedding. Animals were housed at a temperature of $25 \pm 2^{\circ} \mathrm{C}$ and humidity of $30-60 \%$. A $12: 12 \mathrm{~h}$ light and the dark cycle were followed. The animals had free access to food and water ad libitum. All the experimental procedures and protocols used in this study were reviewed by the institutional animal ethics committee (688/2/ Committee for the Purpose of Control and Supervision of Experiments on Animals [CPCSEA]) and were in accordance with the guidelines of the IAEC. Animal care was given as per the guidelines of CPCSEA.

\section{Experimental design}

The animals are divided into four groups $(n=10)$. Experimental diabetes was induced by rats fed with an HFD (58\% calories as fat) for 2 weeks. Then, rats were treated with a single intraperitoneal injection of $35 \mathrm{mg} / \mathrm{kg}$ of STZ [14]. Rats with marked hyperglycemia fasted blood glucose level $>200 \mathrm{mg} / \mathrm{dL}$ were used for the study.

The animals are divided into four groups $(\mathrm{n}=10)$.

- Group I: Normal control (N) 
- $\quad$ Group II: Diabetic rats (HFD+ STZ) (D)

- Group III: Diabetic rats treated with glibenclamide $(600 \mu \mathrm{g} / \mathrm{kg})$ for 14 days

- Group IV: Diabetic rats + glibenclamide + silymarin $50 \mathrm{mg} / \mathrm{kg} / \mathrm{twice} / \mathrm{d}$ for 14 days

Blood samples were collected from the tip of the tail on the $0,4,8,12$, and $15^{\text {th }}$ day after STZ injection by without sacrificing the animals, from the tail vein by snipping off the tip of the tail, and blood glucose was checked by glucometer. On the $30^{\text {th }}$ day, animals were anesthetized and blood samples were collected by retro-orbital puncture into dry nonheparinized tubes for serum biochemical profile, and the serum was separated by centrifuging at $3000 \mathrm{rpm}$ for $15 \mathrm{~min}$.

\section{Preparation of intestinal homogenate}

All rats were sacrificed by cervical dislocation. The intestine was removed, flushed with saline, and washed again. The mucosa was scraped off with the help of a microscopic glass slide [15]. 10\% W/V of the mucosa was mixed with 5 mM EDTA (pH - 7.4) and homogenized with Teflon pestle. The homogenate was centrifuged and the supernatant liquid was used for the estimation of IAP.

\section{Statistical analysis}

The data are expressed as the mean \pm standard deviation. GraphPad Prism software was used to analyze data and construct the graphs. One-way analysis of variance followed by Dunnett's test. The values of $\mathrm{p}<0.05$ were regarded as statistically significant.

\section{RESULTS}

\section{Effect of silymarin on blood glucose}

Blood glucose level of different experimental groups was shown in Table 1. The glucose level was significantly $(\mathrm{p}<0.01)$ increased in diabetic animals compared with normal. The standard drug glibenclamidetreated animals showed significant $(\mathrm{p}<0.01)$ reduction in blood glucose level $(86 \mathrm{mg} / \mathrm{dL})$ when compared with diabetic control. The silymarin and glibenclamide-treated diabetic animals showed $75 \mathrm{mg} / \mathrm{dL}$ glucose on the $15^{\text {th }}$ day of treatment. Silymarin treatment reduces the blood glucose level and used to treat along antidiabetic drugs with caution about hypoglycemia or reduces the dose of an antidiabetic drug.

\section{Effect of silymarin on lipid profile}

Fig. 1 shows the lipid levels of normal and various experimental groups. The diabetic animals showed that cholesterol $(168.9 \mathrm{mg} / \mathrm{dL})$, triglycerides (190.2 mg/dL), LDL (106.1 mg/dL), and VLDL (38 mg/dL) were significantly $(\mathrm{p}<0.01)$ increased when compared to the control. The positive control animals showed significant reduction in cholesterol (164.1 mg/dL), triglycerides (179.7 mg/dL), LDL (101.3 mg/dL), and no significant $(p>0.05)$ difference in VLDL $(35.9 \mathrm{mg} / \mathrm{dL})$ and $\mathrm{HDL}$ $(26.9 \mathrm{mg} / \mathrm{dL})$ when compared to diabetic control. The administration of silymarin along with glibenclamide showed significant $(\mathrm{p}<0.01)$ reduction in cholesterol $(142.5 \mathrm{mg} / \mathrm{dL})$, triglycerides $(121.2 \mathrm{mg} / \mathrm{dL})$, LDL $(65.2 \mathrm{mg} / \mathrm{dL})$, VLDL $(28.5 \mathrm{mg} / \mathrm{dL})$, and increased $(\mathrm{p}<0.05) \mathrm{HDL}$ $(27.5 \mathrm{mg} / \mathrm{dL})$ when compared to diabetic control.

\section{Effect of silymarin on IAP levels}

The diabetic rats showed the significant $(\mathrm{p}<0.01)$ reduction in IAP $(2.82$ $\mathrm{mU} / \mathrm{mg}$ of tissue) levels when compared to control. The antidiabetic drug-treated animals showed $2.94 \mathrm{mU}$ of IAP/mg of tissue. Silymarin and glibenclamide-treated diabetic rats showed $3.36 \mathrm{mU}$ of IAP/mg of tissue. These data suggest that silymarin treatment enhanced the IAP concentrations (Table 2).

\section{DISCUSSION}

T2DM is a metabolic disorder caused by chronic hyperglycemia, primarily occurs in obesity and lack of exercise. The complications of T2DM are cardiovascular complications, eye damage, neuropathy, renal failure, and infections. The vascular complications of T2DM are due to advanced glycated products [16] and primarily by atherosclerosis.

Studies were reported IAP levels that are low in T2DM patients, high IAP levels prevent the development of T2DM even though if the person is in obese, deficiency of IAP can increase the incidence of T2DM [17]. The new therapeutic approach might be involving upregulation of IAP by small molecules such as curcumin, omega-3 fatty acid, and corn oil [18-20]. Silymarin is an herbal drug with antioxidant and antiinflammatory properties; it improves glycemic control and prevents the progression of the diabetic complications. The silymarin mechanisms of action are not fully understood. Silymarin possesses antioxidant activity. It inhibits lipid peroxidation [21,22], prevents glutathione depletion [23], and activates antioxidant enzymes that protect DNA from degradation [24]. Silymarin administration protects the kidney from diabetic damage [25].

In our study showed that silymarin administration decreases the blood glucose level in diabetic rats. Our results are consistent with Mohamed et al. who reported blood glucose of silymarin-treated group was significantly decreased when compared to initial blood glucose [26]. Antidiabetic drug glibenclamide coadministered with silymarin showed good glycemic control and chances of hypoglycemia. Hence,

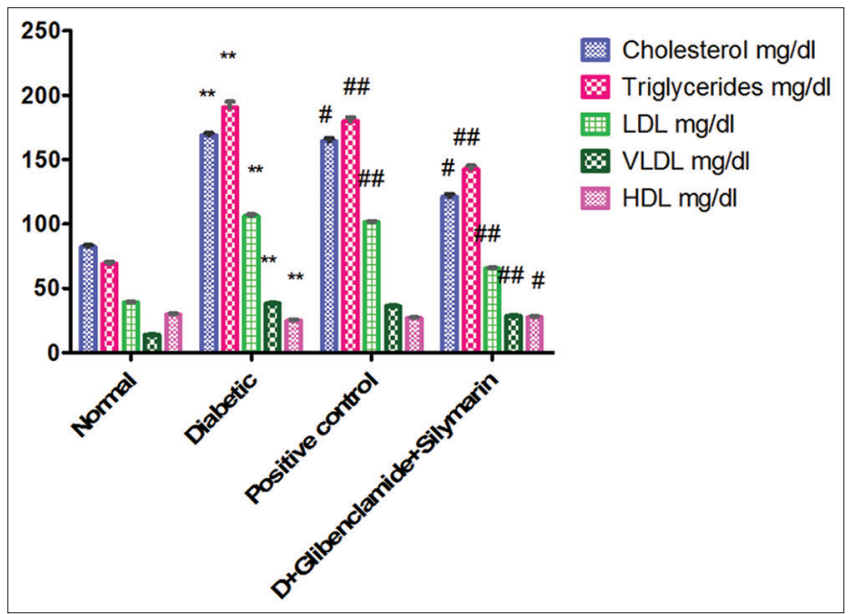

Fig. 1: Lipid profile in different experimental groups. Values are mean \pm standard deviation; $\mathbf{n}=10$ in each group; ${ }^{* *} \mathbf{p}<0.01$ when compared to normal control. ${ }^{\#} \mathbf{p}<0.05$ and ${ }^{\# \#} p<0.01$ when compared to diabetic control (one-way ANOVA followed by Dunnett's test)

Table 1: Blood glucose levels in different experimental groups

\begin{tabular}{|c|c|c|c|c|c|}
\hline \multirow[t]{2}{*}{ Groups } & \multicolumn{5}{|c|}{ Blood glucose in $\mathrm{mg} / \mathrm{dL}$} \\
\hline & $0^{\text {th }}$ day & $4^{\text {th }}$ day & $8^{\text {th }}$ day & $12^{\text {th }}$ day & $15^{\text {th }}$ day \\
\hline Normal & $98 \pm 2.6$ & $102 \pm 3.2$ & $96 \pm 3.6$ & $101 \pm 4.1$ & $104 \pm 3.5$ \\
\hline Diabetic (D) & $232 \pm 4.8^{* *}$ & $243 \pm 4.6^{* *}$ & $248 \pm 2.8^{* *}$ & $236 \pm 3.7^{* *}$ & $231 \pm 2.9^{* *}$ \\
\hline Positive control & $228 \pm 3.2^{\mathrm{ns}}$ & $146 \pm 2.8^{\mathrm{aa}}$ & $133 \pm 4.3^{\text {aа }}$ & $98 \pm 1.9^{\text {aа }}$ & $86 \pm 2.2^{\mathrm{aa}}$ \\
\hline D+glibenclamide+silymarin & $236 \pm 2.9^{\mathrm{ns}}$ & $140 \pm 3.6^{\text {aa }}$ & $114 \pm 2.7^{\text {aa }}$ & $88 \pm 3.1^{\text {aa }}$ & $75 \pm 3.4^{\text {aa }}$ \\
\hline
\end{tabular}

Values are mean \pm SD; $n=10$ in each group; ${ }^{* *} \mathrm{p}<0.01$ when compared to normal control. ${ }^{\text {ns }} \mathrm{p}>0.05$ and ${ }^{\text {aa }} \mathrm{p}<0.01$ when compared to diabetic control (one-way ANOVA

followed by Dunnett's test), SD: Standard deviation 
Table 2: IAP levels in control and experimental groups

\begin{tabular}{ll}
\hline Groups & IAP $\mathbf{~ m U} / \mathbf{m g}$ of tissue \\
\hline Normal & $4.49 \pm 0.08$ \\
Diabetic (D) & $2.82 \pm 0.04^{* *}$ \\
D+glibenclamide & $2.94 \pm 0.06^{\mathrm{ns}}$ \\
D+glibenclamide+silymarin & $3.36 \pm 0.05^{\mathrm{aa}}$ \\
\hline
\end{tabular}

Values are mean $\pm \mathrm{SD} ; \mathrm{n}=10$ in each group; ${ }^{* *} \mathrm{p}<0.01$ when compared to normal control. ${ }^{\text {ns }} \mathrm{p}>0.05$ and ${ }^{\text {aa }} \mathrm{p}<0.01$ when compared to diabetic control (one-way ANOVA followed by Dunnett's test), IAP: Intestinal alkaline phosphatase,

SD: Standard deviation

we need to monitor the blood glucose level and reduce the dose of glibenclamide.

Silymarin-treated rats showed decreased total cholesterol, triglyceride, VLDL, LDL, and increased HDL levels. Silymarin decreases the VLDL synthesis and the availability of free VLDL secretion in the intestine. The IAP binds to intracellular proteins and decreases the movement of the fat particles across the enterocyte. The IAP decreases fat absorption in animals that ingest HFDs [27].

Deficiency of IAP can increase the incidence of T2DM, increase fat absorption, increase intestinal inflammation, alter gut integrity, and enhance the penetration of microbes [13]. Exogenous human recombinant IAP administration protects against this hyperlipidemia, diabetes mellitus, and metabolic syndrome and their complications [28]. The chronic administration of IAP is required to avoid such complications, but the IAP is costly, we searched a herbal remedy with low cost and for an alternate for IAP. Silymarin was already reported for the treatment of diabetes mellitus and hyperlipidemia. However, our study showed that silymarin acts on intestine and increases the expression of IAP and protects against incidence and progression of T2DM and its complications. In case of both Type 1 and T2DM, it may reduce the vascular complications by decrease the fat absorption.

\section{CONCLUSION}

The present study clearly concluded that the silymarin increased the IAP level in the intestine. Silymarin is the cheap herbal remedy to prevent incidence and progression of T2DM. Further studies are required to analyze the role of silymarin in T1DM complications.

\section{AUTHORS' CONTRIBUTION}

Both authors were contributed equally to the design and implementation of the research, analysis of the results, and writing of the manuscript.

\section{CONFLICTS OF INTEREST}

The authors declare that they have no conflicts of interest.

\section{REFERENCES}

1. Weyer C, Bogardus C, Mott DM, Pratley RE. The natural history of insulin secretory dysfunction and insulin resistance in the pathogenesis of Type 2 diabetes mellitus. J Clin Invest 1999;104:787-94.

2. Lebovitz HE. Diagnosis, classification, and pathogenesis of diabetes mellitus. J Clin Psychiatry 2001;62 Suppl 27:5-9.

3. American Diabetes Association. Diagnosis and classification of diabetes mellitus. Diabetes Care 2014;37 Suppl 1:S81-90.

4. LeRoith D, Fonseca V, Vinik A. Metabolic memory in diabetes - focus on insulin. Diabetes Metab Res Rev 2005;21:85-90.

5. Abdullah N, Attia J, Oldmeadow C, Scott RJ, Holliday EG. The architecture of risk for Type 2 diabetes: Understanding Asia in the context of global findings. Int J Endocrinol 2014;2014:21.

6. Comelli MC, Mengs U, Schneider C, Prosdocimi M. Toward the definition of the mechanism of action of silymarin: Activities related to cellular protection from toxic damage induced by chemotherapy. Integr Cancer Ther 2007;6:120-9.

7. Boigk G, Stroedter L, Herbst H, Waldschmidt J, Riecken EO, Schuppan D, et al. Silymarin retards collagen accumulation in early and advanced biliary fibrosis secondary to complete bile duct obliteration in rats. Hepatology 1997;26:643-9.

8. Hassanein H, Abdel-Mohsen M, Shams K, Abdel-Azim N, Saleh M, Ehsan N, et al. Comparative analysis of the biological activity of Silybum marianum L. Food supplements available on the market: In vitro study. Int J Pharm Pharm Sci 2015;7:55-60.

9. Suh HJ, Cho SY, Kim EY, Choi HS. Blockade of lipid accumulation by silibinin in adipocytes and zebrafish. Chem Biol Interact 2015;227:53- 62 .

10. Van Dongen JM, Kooyman J, Visser WJ, Holt SJ, Galjaard H. The effect of increased crypt cell proliferation on the activity and subcellular localization of esterases and alkaline phosphatase in the rat small intestine. Histochem J 1977;9:61-75.

11. Bates JM, Mittge E, Kuhlman J, Baden KN, Cheesman SE, Guillemin K, et al. Distinct signals from the microbiota promote different aspects of zebrafish gut differentiation. Dev Biol 2006;297:374-86.

12. Lallès JP. Intestinal alkaline phosphatase: Novel functions and protective effects. Nutr Rev 2014;72:82-94.

13. Jason F, David G. Intestinal alkaline phosphatase: A summary of its role in clinical disease. J Surg Res 2016;202:225-34.

14. Srinivasan K, Viswanad B, Asrat L, Kaul CL, Ramarao P. Combination of high-fat diet-fed and low-dose streptozotocin-treated rat: A model for Type 2 diabetes and pharmacological screening. Pharmacol Res 2005;52:313-20.

15. Takanori K, Tomoyuki T, Shuhachi K, Kei S. Facile preparation of rat intestinal mucosa for assay of mucosal enzyme activity. J Nutr Sci Vitaminol 1993;39:399-403.

16. Su-Yen G, Mark EC. The role of advanced Glycation end products in progression and complications of diabetes. J Clin Endocrinol Metabol 2008;93:1143-52.

17. Madhu SM. A high level of intestinal alkaline phosphatase is protective against Type 2 diabetes mellitus irrespective of obesity. EBioMedicine 2015;2:2016-23.

18. Ghosh SS, Gehr TW, Ghosh S. Curcumin and chronic kidney disease (CKD): Major mode of action through stimulating endogenous intestinal alkaline phosphatase. Molecules 2014;19:20139-56.

19. Kaliannan K, Wang B, Li XY, Kim KJ, Kang JX. A host-microbiome interaction mediates the opposing effects of omega- 6 and omega-3 fatty acids on metabolic endotoxemia. Sci Rep 2015;5:11276.

20. Mahmood A, Shao JS, Alpers DH. Rat enterocytes secrete SLPs containing alkaline phosphatase and cubilin in response to corn oil feeding. Am J Physiol Gastrointest Liver Physiol 2003;285:G433-41.

21. Bosisio E, Benelli C, Pirola O. Effect of the flavanolignans of silybum marianum L. On lipid peroxidation in rat liver microsomes and freshly isolated hepatocytes. Pharmacol Res 1992;25:147-54.

22. Wellington K, Adis BJ. Silymarin: A review of its clinical properties in the management of hepatic disorders. BioDrugs 2001;15:465-89.

23. Tasduq SA, Peerzada K, Koul S, Bhat R, Johri RK. Biochemical manifestations of anti-tuberculosis drugs induced hepatotoxicity and the effect of silymarin. Hepatol Res 2005;31:132-5.

24. Kiruthiga PV, Shafreen RB, Pandian SK, Devi KP. Silymarin protection against major reactive oxygen species released by environmental toxins: Exogenous $\mathrm{H}_{2} \mathrm{O}_{2}$ exposure in erythrocytes. Basic Clin Pharm Toxicol 2007;100:414-9.

25. Khazim K, Gorin Y, Cavaglieri RC, Abboud HE, Fanti P. The antioxidant silybin prevents high glucose-induced oxidative stress and podocyte injury in vitro and in vivo. Am J Physiol Renal Physiol 2013;305:F691- 700.

26. Mohamed EF, Amr N, Amira AE, Mona W. Antioxidant therapeutic actions of medical phytochemicals, silymarin, and silibinin on streptozotocin diabetic rats: First novel comparative assessment of structural recoveries of histological and ultrastructural changes on islets of Langerhans. Int J Pharm Pharm Sci 2016;8:69-76.

27. Sonoko N, Lei H, Aratr I, Hideaki H, David HA, Jose LM. Accelerated fat absorption in intestinal alkaline phosphatase knockout mice. Molecular Cellular Biol 2003;23:7525-30.

28. Kaliannan K, Hamarneh SR, Economopoulos KP, Nasrin Alam S, Moaven O, Patel $\mathrm{P}$, et al. Intestinal alkaline phosphatase prevents metabolic syndrome in mice. Proc Natl Acad Sci U S A 2013;110:7003-8. 